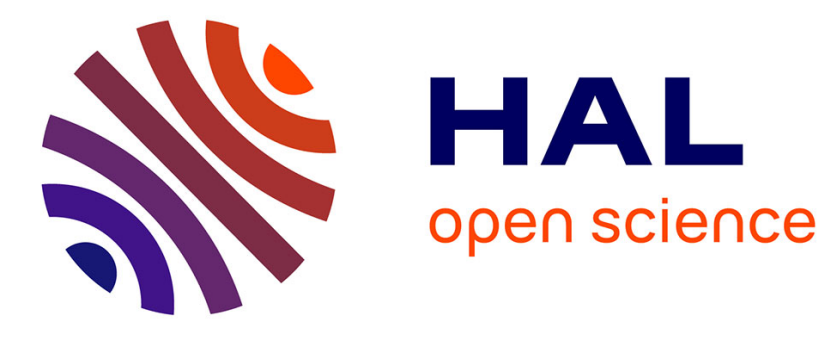

\title{
Analyse du processus de formation de la barrière or-silicium
}

G. Pananakakis, P. Viktorovitch, J.P. Ponpon

\section{To cite this version:}

G. Pananakakis, P. Viktorovitch, J.P. Ponpon. Analyse du processus de formation de la barrière or-silicium. Revue de Physique Appliquée, 1978, 13 (9), pp.449-455. 10.1051/rphysap:01978001309044900 . jpa-00244474

\section{HAL Id: jpa-00244474 https://hal.science/jpa-00244474}

Submitted on 1 Jan 1978

HAL is a multi-disciplinary open access archive for the deposit and dissemination of scientific research documents, whether they are published or not. The documents may come from teaching and research institutions in France or abroad, or from public or private research centers.
L'archive ouverte pluridisciplinaire HAL, est destinée au dépôt et à la diffusion de documents scientifiques de niveau recherche, publiés ou non, émanant des établissements d'enseignement et de recherche français ou étrangers, des laboratoires publics ou privés. 


\title{
ANALYSE DU PROCESSUS DE FORMATION DE LA BARRIËRE OR-SILICIUM
}

\author{
G. PANANAKAKIS, P. VIKTOROVITCH \\ Laboratoire d'Electronique, E.R.A. no ${ }^{659}$, E.N.S.E.R.G., \\ 23, avenue des Martyrs, 38031 Grenoble-Cedex, France \\ et J. P. PONPON \\ Centre de Recherches Nucléaires, Groupe de Physique et Applications des Semiconducteurs, \\ 67037 Strasbourg-Cedex, France
}

(Reçu le 10 février 1978, révisé le 17 mai 1978, accepté le 22 mai 1978)

\begin{abstract}
Résumé. - Nous comparons les caractéristiques courant-tension sous éclairement de diodes Schottky or-silicium à des courbes théoriques de structures MIS. Nous montrons que l'évolution dans le temps de ces caractéristiques est due à la neutralisation d'une charge fixe positive, située à l'interface métal-semiconducteur, par l'oxygène de l'air qui diffuse à travers le contact métallique et qui se comporte en piège à électrons du semiconducteur.
\end{abstract}

\begin{abstract}
We compare the experimental current-voltage characteristics of gold-silicon Schottky diodes with theoretical curves of MIS structures. We show that the ageing process results from the neutralization of a fixed positive charge at the metal-semiconductor interface, due to oxygen from the ambiant diffusing through the rectifying contact. It is further demonstrated that oxygen behaves like a semiconductor electron trap, which explains the decrease of the neutralization efficiency during the barrier formation and the locking of the photovoltage.
\end{abstract}

1. Introduction. - Les cellules solaires de type structure MIS de Schottky ont connu récemment un essor très important, qui a fait progresser leur rendement de conversion photovoltaïque au-delà de $10 \%$ dans le cas' du silicium monocristallin. Parallèlement aux études expérimentales, la mise au point de modèles de fonctionnement de ces dispositifs a permis de déterminer les paramètres dont l'influence est prépondérante et ainsi de préciser les conditions expérimentales requises pour une optimisation des performances [1-4]. Cependant, une instabilité dans le temps des propriétés de ces dispositifs a été observée [5], ce qui a conduit à reconsidérer le problème $\mathrm{du}$ vieillissement des diodes Schottky, mis en évidence il y a plus de dix ans [6,7]. Dans un article précédent [8], nous avons fait apparaître que le vieillissement des structures or-silicium de type $\mathbf{N}$ est directement liéà la formation de la barrière de potentiel et dû à la diffusion, à travers le film d'or, d'oxygène ou de vapeur d'eau qui s'accumule à l'interface.

Le but de cet article est de montrer que l'utilisation conjointe d'un modèle théorique du comportement des structures MIS et de résultats expérimentaux sur l'évolution temporelle des diodes or-silicium peut conduire à une meilleure compréhension du phénomène de formation de la barrière de potentiel au contact métal-semiconducteur.

2. Résultats expérimentaux. -2.1 TeChNiQue EXPéRIMENTALE. - Les mesures sont effectuées sur du silicium de type $N$, de basse résistivité (la concentration de porteurs est comprise entre 1 et $5 \times 10^{20} \mathrm{~m}^{-3}$ ). Après rodage, les plaquettes sont munies sur leur face arrière d'un contact ohmique réalisé par dépôt de $300 \AA$ Å d'aluminium. La face avant est ensuite décapée chimiquement dans une solution de CP4. Après rinçage à l'eau désionisée et, éventuellement à l'acide fluorhydrique dilué, les échantillons sont placés sous vide (pression résiduelle inférieure à $5 \times 10^{-6}$ torr) et un film d'or est déposé par évaporation. L'évolution dans le temps des propriétés des dispositifs est étudiée par des mesures électriques (relevé des caractéristiques courant-tension sous un éclairement d'environ $100 \mathrm{~mW} / \mathrm{cm}^{2}$ fourni par une lampe quartzhalogène reproduisant grossièrement le spectre solaire 
et détermination de la hauteur de la barrière de potentiel par la mesure de courant de saturation et par la réponse photoélectrique) et par l'analyse de la distribution d'oxygène à l'interface or-silicium (par spectroscopie de masse d'ions secondaires et par la réaction nucléaire $\left.{ }^{16} \mathrm{O}\left(\alpha, \alpha^{\prime}\right)^{16} \mathrm{O}\right)$.

2.2 CARACTÉRISTIQUeS COURANT-TENSION. - La figure $1 a$ représente, pour un échantillon possédant un film d'or de $300 \AA$ d'épaisseur, les caractéristiques courant-tension sous éclairement, relevées sous vide après dépôt du métal et pour des temps relativement courts après mise à l'air. L'évolution de ces courbes pour des temps très longs après la mise à l'air est montrée sur la figure $1 b$. Sur la figure $1 c$, nous avons reporté des caractéristiques similaires correspondant à

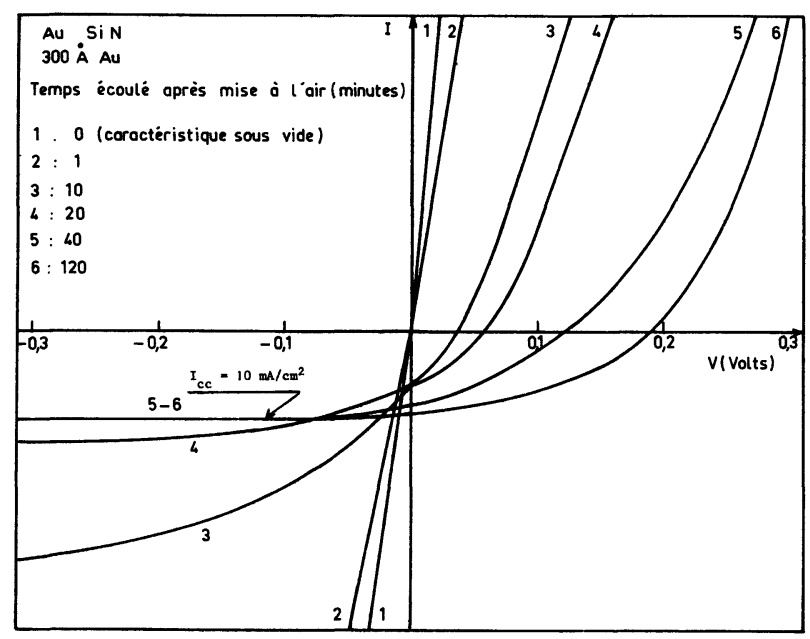

Fig. 1a. - Evolution temporelle (mise à l'air de $120 \mathrm{~min}$.) des caractéristiques courant-tension sous illumination pour un échantillon or-silicium de type $\mathbf{N}$ (épaisseur de la couche d'or de $300 \AA$ ).

[Evolution with time (up to $120 \mathrm{~min}$. of exposure to air) of the current-voltage characteristics under illumination for $300 \AA$ gold on N-type silicon.]

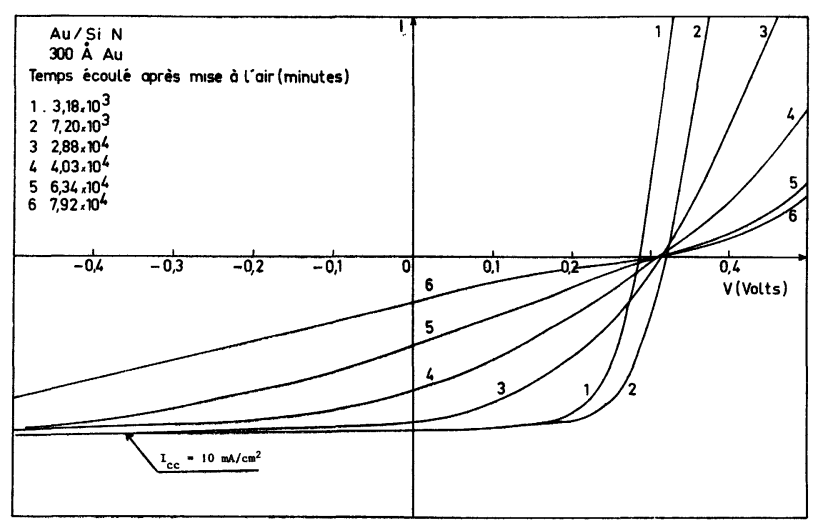

FIG. $1 b$. - Evolution pour des temps plus longs de mise à l'air des caractéristiques de l'échantillon de la figure $1 a$. L'état optimum (courbe 2) est atteint $10^{4} \mathrm{~min}$. environ après la mise à l'air.

[Evolution for longer times of exposure to air of the characteristics of the sample of figure $1 a$. The optimum (curve 2) is reached after about $10^{4} \mathrm{~min}$.]

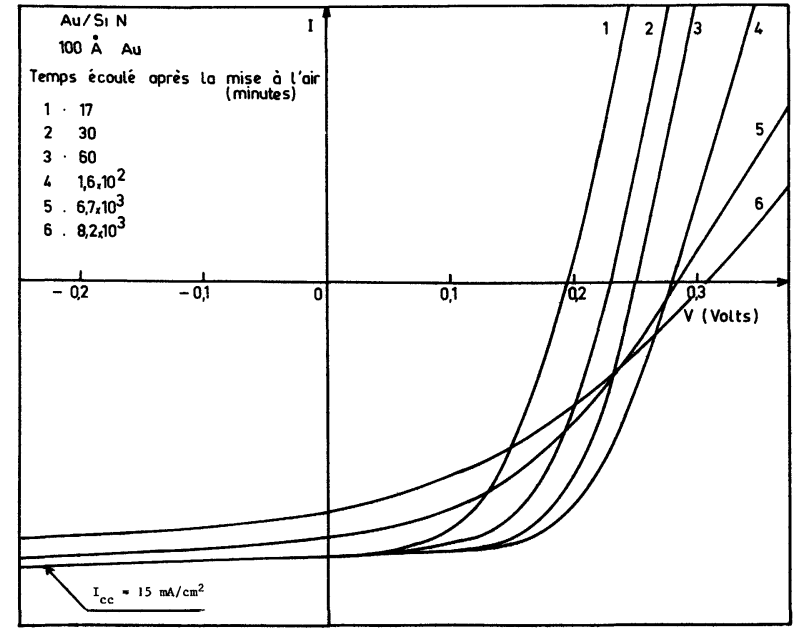

Fig. 1c. - Evolution temporelle (mise à l'air de $6700 \mathrm{~min}$.) des caractéristiques courant-tension sous illumination pour un échantillon or-silicium de type $\mathrm{N}$ (épaisseur de la couche d'or de $100 \AA$ ).

[Evolution with time (up to $6700 \mathrm{~min}$. of exposure to air) of the current-voltage characteristics under illumination for $100 \AA$ gold on N-type silicon.]

l'évolution d'un échantillon possédant un film d'or de $100 \AA$ d'épaisseur et dans un domaine de temps plus restreint. On constate que, tant que le dispositif demeure sous vide, il se comporte comme une résistance (pas de redressement) et ne délivre pas, ou presque pas, de tension photovoltaïque. Lorsqu'il est mis à l'air, une caractéristique de redressement et une tension photovoltaique apparaissent progressivement après un temps d'attente qui dépend essentiellement de l'épaisseur de la couche d'or. Alors que ce temps est très bref pour $100 \AA$ d'or (quelques dizaines de secondes), il est beaucoup plus long pour $300 \AA$ (quelques minutes). La tension photovoltaïque croît jusqu'à la valeur de saturation, de l'ordre de $0,3 \mathrm{~V}$, qui est atteinte en un temps dépendant de l'épaisseur du métal et du degré hygrométrique. Pour des temps d'attente beaucoup plus importants, le phénomène de suppression du courant photovoltaïque se manifeste (Fig. 1b), la tension ne changeant pratiquement pas, en atmosphère sèche tout au moins.

2.3 HaUteur DE LA BarRière DE POTENTIEL. - Les résultats fournis par les deux types de mesures utilisées pour la détermination de la hauteur de barrière sont en bon accord, aux erreurs expérimentales près, soit $0,03 \mathrm{eV}$. Nous constatons que la barrière de potentiel $\varphi_{\mathrm{Bn}}$ est initialement assez faible $(0,5$ à $0,6 \mathrm{eV})$ et qu'elle augmente, en fonction du temps que l'échantillon passe à l'air (après un temps d'attente qui varie selon l'épaisseur du film d'or) jusqu'à la valeur caractéristique du contact or-silicium de type $\mathrm{N}$, soit 0,80 à $0,83 \mathrm{eV}[8]$.

2.4 DisTRIBUTION DE L'OXYGÈNE A L'INTERFACE. L'analyse de l'évolution de la distribution de l'oxygène 
à l'interface ayant déjà été publiée $[8,9]$, nous en rappellerons seulement les résultats essentiels. Nous avons montré que, dès que les échantillons sont mis à l'air, l'oxygène de l'air ambiant diffuse à travers le film d'or (la constante de diffusion a été trouvée égale à $8,4 \times 10^{-20} \mathrm{~m}^{2} / \mathrm{s}$ ) puis s'accumule à l'interface, la dépendance temporelle de ce dernier processus étant logarithmique, à un taux de $1,2 \times 10^{19} \mathrm{at} / \mathrm{m}^{2}$ par décade. D'autre part, les propriétés électriques des dispositifs sont étroitement liées à la présence d'oxygène, un accroissement de la concentration de ce dernier à l'interface d'environ $2 \times 10^{19} \mathrm{~m}^{-2}$ étant nécessaire pour obtenir la saturation de la tension photovoltaïque et la valeur typique de $\varphi_{\mathrm{Bn}}$.

3. Analyse des résultats expérimentaux. - Nous interprétons ici les résultats expérimentaux précédents en résolvant numériquement les équations fondamentales du fonctionnement des structures MIS. Ces équations ont été établies et discutées dans des publications antérieures $[1,2]$; seules seront rapportées les modifications introduites afin de tenir compte des particularités présentées par les structures étudiées.

Nous pouvons distinguer deux phases d'évolution des structures examinées. La première phase commence après la mise à l'air des échantillons jusqu'à l'établissement de la tension de circuit ouvert $V_{\text {oc }}$ maximum et l'optimisation des paramètres de l'échantillon. La seconde phase correspond à des temps très longs après la mise à l'air (période de vieillissement de la structure) et est caractérisée par l'apparition de l'effet de suppression de courant et par le maintien de la tension $V_{\text {oc }}$.

3.1 PRemière PHASE. - La détérioration des propriétés de redressement et photovoltaïques d'une

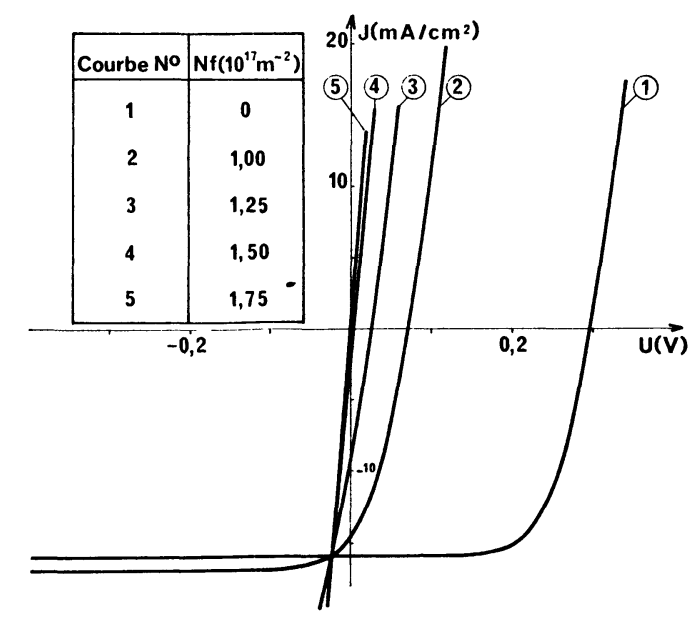

FIG. 2. - Influence d'une charge positive à l'interface isolantsemiconducteur sur les caractéristiques $J-V$ d'une structure MIS avec $\chi_{\mathrm{n}}=\chi_{\mathrm{p}}=0,5 \mathrm{eV}$ (pour les autres paramètres de la structure voir annexe A.2).

[Influence of a positive charge located at the insulator-semiconductor interface on $J-V$ characteristics of a MIS structure (with $\chi_{\mathrm{n}}=\chi_{\mathrm{p}}=0.5 \mathrm{eV}$ ) (for other parameters see annex A2).] structure MIS peut être attribuée à l'existence de charges positives situées à l'interface semiconducteurisolant et à son voisinage $[2,3,10]$. Pour une densité donnée de ces charges, qui peuvent correspondre aux défauts du réseau cristallin, l'effet est d'autant plus important que l'épaisseur de la couche isolante est plus grande.

Nous avons donc introduit dans l'expression de la tension supportée par la couche isolante, $U_{\mathrm{s}}$, une densité de charge $Q_{\mathrm{f}}$ (par unité de surface), correspondant à une densité de charges élémentaires $N_{\mathrm{f}}$, définie par l'expression :

$$
Q_{\mathrm{f}}=N_{\mathrm{f}} \cdot q
$$

par la suite nous utiliserons le terme charge à la place de densité de charge.

Nous avons étudié l'influence de ces charges sur les propriétés électriques de la structure, avec $N_{\mathrm{f}}$ comme paramètre pour des valeurs de l'épaisseur de la couche isolante $\delta$ compatibles avec le traitement physico-chimique des échantillons. Les résultats ainsi obtenus (Fig. 2) reproduisent correctement le réseau expérimental de la figure $1 a$ pour $\delta=5 \AA$ et

$$
0 \leqslant N_{\mathrm{f}} \leqslant 1,8 \times 10^{17} / \mathrm{m}^{2} .
$$

Il en résulte que la structure atteint son état optimum lorsque toute la charge $N_{\mathrm{f}}$ se trouve neutralisée.

3.2 DeuXième PhaSe. - L'effet de suppression de courant caractérisant cette période peut être attribué, soit à l'existence d'une résistance parasite $R_{\mathrm{s}}$ en série avec la structure MIS idéale, soit à la présence d'une couche isolante trop épaisse, réduisant la collecte des porteurs minoritaires [4]. L'action de pièges ayant un rôle cinétique (effet de génération-recombinaison à l'interface) est à exclure car elle n'affecte pas la valeur du courant de court-circuit de la structure comme nous l'avons montré dans [4].

La présence d'une résistance série parasite est exprimée par la relation :

$$
U=\delta V_{\mathrm{s}}+\delta U_{\mathrm{s}}+V_{\mathrm{sc}}+R_{\mathrm{s}} J,
$$

où $U$ est la tension extérieure aux bornes de la structure, $J$ la densité de courant, $V_{\text {sc }}$ la chute de tension ohmique dans le volume du semiconducteur. $\delta V_{\mathrm{s}}$, $\delta U_{\mathrm{s}}$ sont les variations de la barrière de surface du semiconducteur et de la tension $U_{\mathrm{s}}$ par rapport à leurs valeurs à l'équilibre thermodynamique.

L'influence d'une résistance série parasite sur les caractéristiques $J-V$ de la structure est montrée par le réseau de courbes de la figure 3 . Bien que la suppression du courant s'accompagne du maintien de $V_{\text {oc }}$, le désaccord avec les courbes expérimentales ne permet pas de retenir cette hypothèse. En effet, en présence d'une résistance série importante, les caractéristiques sont assimilables à des droites, contrairement à ce que l'on observe sur la figure $1 b$. L'effet de suppression du courant photovoltaïque peut, par conséquent, être 


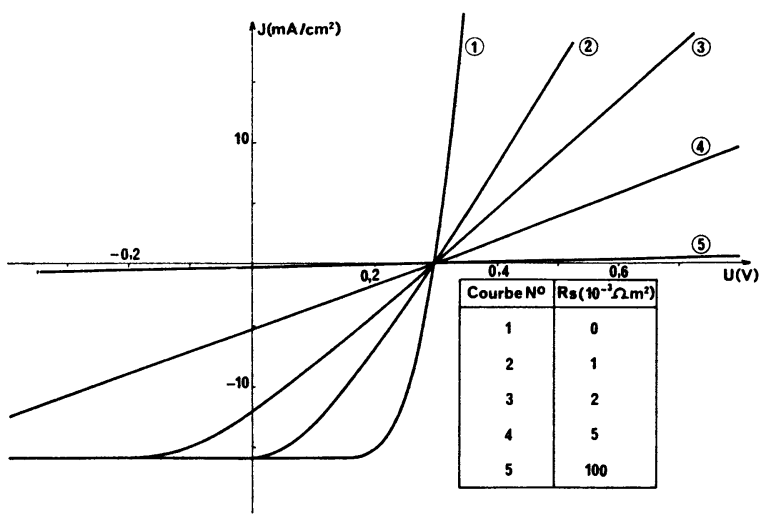

FIG. 3. - Effet d'une résistance $R_{\mathrm{s}}$ en série avec la structure MIS idéale.

[Effect of a series resistance.]

expliqué par l'augmentation de l'épaisseur de la couche isolante. Cependant, si l'on admet pour l'affinité des électrons et des trous, les valeurs utilisées habituellement, soit $\chi_{\mathrm{n}}=0,5 \mathrm{eV}$ et $\chi_{\mathrm{p}}=0,5 \mathrm{eV}$, on constate que, d'après les résultats exposés dans [1] la tension $V_{\text {oc }}$ doit croître avec $\delta$ (pour $\delta$ compris entre 0 .et $25 \AA$ ), ce qui est en désaccord avec les résultats expérimentaux. Cette contradiction peut être levée car la hauteur de la barrière $\varphi_{\mathrm{Bn}}$ de la structure est la même, quelle que soit la méthode expérimentale utilisée (optique ou électrique). Ceci signifie que la couche isolante est transparente pour les porteurs majoritaires (électrons); en d'autres termes la probabilité de passage des électrons à travers la couche isolante est strictement égale à l'unité. Il faut donc conclure que l'isolant ne présente aucune barrière pour les électrons et leur affinité $\chi_{n}$ est négative ou nulle. En tenant compte de la forme des caractéristiques expérimentales en polarisation inverse et des résultats de [11] on peut estimer que la valeur de l'affinité de $\chi_{\mathrm{p}}$ adaptée à la structure étudiée est égale à $2 \mathrm{eV}$. Il est alors possible de proposer un schéma qualitatif de la structure des bandes des échantillons MIS en question (Fig. 4). Notons qu'un tel schéma

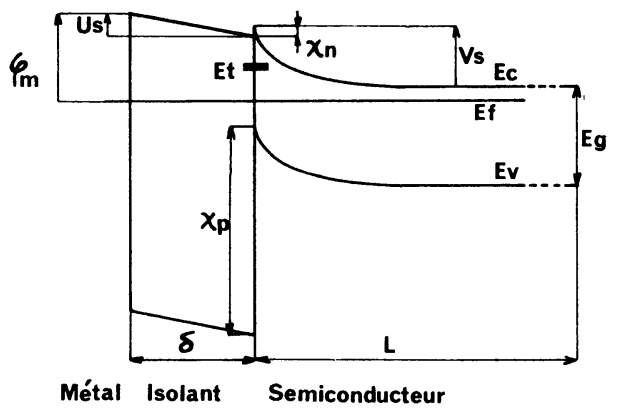

FIG. 4. - Structures de bandes d'énergie des échantillons MIS étudiés.

[Band structure of the MIS diode.] présente un cảractère formel dans la mesure où il est délicat d'attribuer une structure de bandes à une couche isolante inférieure à $20 \AA$.

3.3 EFFETS De PIÉGEAGE. - En utilisant les valeurs des affinités des électrons et des trous que nous avons déterminées plus haut, soit $\chi_{\mathrm{n}}=0$ et $\chi_{\mathrm{p}}=2 \mathrm{eV}$, nous constatons par le calcul que la tension $V_{\text {oc }}$ de la structure reste pratiquement égale à sa valeur expérimentale $(300 \mathrm{mV})$ pour $0 \leqslant \delta \leqslant 10 \AA$. Au-delà de cette épaisseur, $V_{\mathrm{oc}}$ diminue (Fig. 5 ). Cette dernière contradiction avec l'expérience nous incite à penser à l'existence de pièges de type accepteur situés en énergie plus près de la bande de conduction que de la bande de valence et interagissant avec les électrons du semiconducteur [1]. Outre l'amélioration de $V_{\text {oc }}$ sans modification du courant de court-circuit $J_{\mathrm{cc}}$ et de la partie en polarisation inverse des caractéristiques $J-V$, ce type de pièges provoque l'augmentation du facteur d'idéalité de la structure, ce qui est en parfait accord avec l'expérience (Fig. 1b).

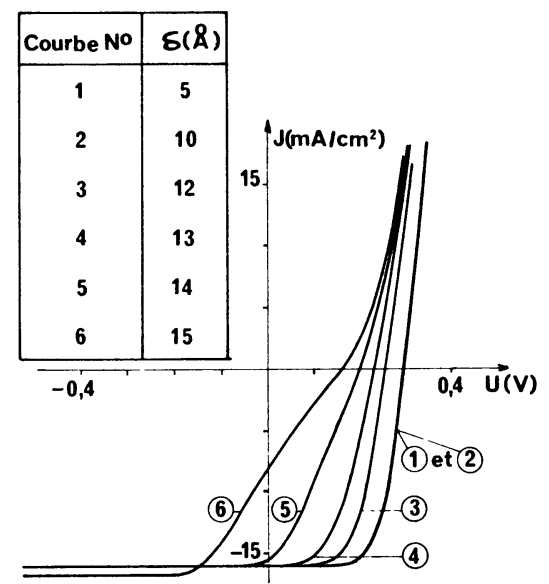

Fig. 5. - Caractéristiques $J-V$ d'une structure MIS avec $\chi_{\mathrm{n}}=0$ et $\chi_{\mathrm{p}}=2 \mathrm{eV}$, en l'absence de pièges à l'interface.

$\left[J-V\right.$ characteristics of a MIS structure for $\chi_{\mathrm{n}}=0$ and $\chi_{\mathrm{p}}=2 \mathrm{eV}$, without interface traps.]

$\mathrm{Ne}$ disposant pas d'informations expérimentales directes concernant la nature des pièges à l'interface isolant-semiconducteur nous avons considéré le cas le plus simple susceptible de fournir des résultats en accord avec les courbes expérimentales. C'est ainsi que nous avons introduit la charge des pièges ionisés $Q_{\mathrm{ss}}$ exprimée par la fonction de Fermi :

$$
Q_{\mathrm{ss}}=-\frac{q D_{\mathrm{ss}}}{1+\exp \frac{E_{\mathrm{t}}-E_{\mathrm{Fn}}}{k T}} ;
$$

$E_{\mathrm{t}}, E_{\mathrm{Fn}}$ désignant respectivement le niveau énergétique des pièges (supposés monoénergétiques) et le quasi-niveau de Fermi des électrons du semiconducteur. $D_{\mathrm{ss}}$ est la densité de pièges. 
Le réseau de caractéristiques de la figure 6 , obtenu pour $E_{\mathrm{t}}$ situé à $0,425 \mathrm{eV}$ sous la bande de conduction et $D_{\text {ss }}$ égal à $10^{17} / \mathrm{m}^{2}$ pour $\delta$ compris entre 0 et $10 \AA$ et à $2 \times 10^{19} / \mathrm{m}^{2}$ pour $\delta$ égal à $15 \AA$, reproduit les résultats expérimentaux de la figure $1 b$. Il est à noter que l'on obtient des résultats identiques si l'on adopte une valeur constante pour $D_{\text {ss }}$ et des valeurs variables pour la position du niveau $E_{\mathrm{t}}$ par rapport aux bandes d'énergie ou inversement si l'on considère que $E_{\mathrm{t}}$ est constant et $D_{\text {ss }}$ variable.

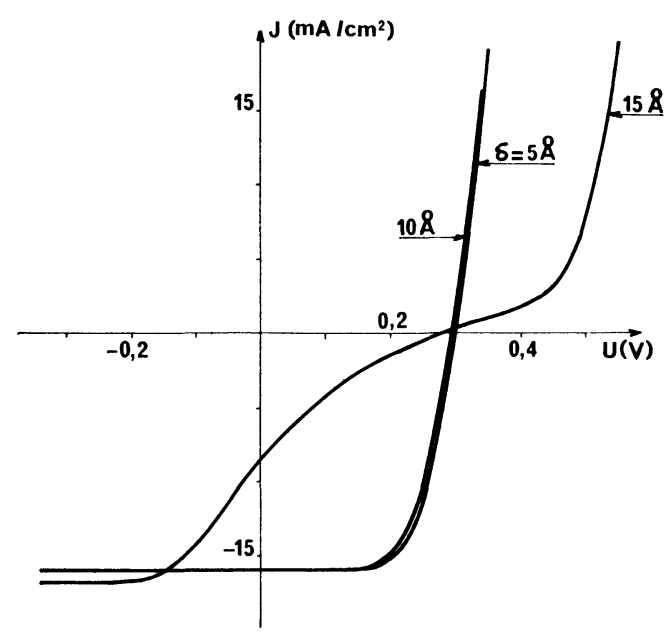

Fig. 6. - Caractéristiques $J-V$ reproduisant le cas de la figure 5 mais en présence de pièges monoénergétiques de niveau $E_{\mathrm{t}}$ situés à $0,425 \mathrm{eV}$ de la bande de conduction et de densité : I) $D_{\mathrm{ss}}=10^{17} / \mathrm{m}^{2}$ pour $0 \leqslant \delta \leqslant 10 \AA$; II) $D_{\mathrm{ss}}=2 \times 10^{19} / \mathrm{m}^{2}$ pour $\delta=15 \AA$.

[Theoretical $J-V$ characteristics in presence of single energy $E_{\mathrm{t}}$ interface traps ; $E_{\mathrm{t}}$ is located at $0.425 \mathrm{eV}$ below the conduction band edge : I) $D_{\mathrm{ss}}=10^{17} / \mathrm{m}^{2}$ for $0 \leqslant \delta \leqslant 10 \AA$; II) $D_{\mathrm{ss}}=2 \times 10^{19} / \mathrm{m}^{2}$ for $\delta=15 \AA$.

3.4 RÔLE DE L'OXYGÈNE. - L'effet du piégeage lors de la deuxième phase d'évolution des dispositifs a été discuté dans le paragraphe précédent. Cependant, l'exploitation conjuguée des résultats théoriques et expérimentaux concernant la phase de neutralisation (première phase d'évolution) permet de montrer que le piégeage se manifeste également durant cette période. A l'aide des figures $1 a, b, c$ et en tenant compte des erreurs de mesures relatives aux faibles valeurs de $V_{\text {oc }}$ et à l'évolution de l'épaisseur $\delta$ de la couche isolante, nous pouvons déterminer un réseau de courbes qui représente l'évolution temporelle de la charge neutralisée à l'interface (Fig. 7).

Afin de comparer la quantité de charge neutralisée avec la concentration d'oxygène en excès $\Delta[\mathrm{O}]$, accumulé à l'interface, nous avons reporté sur cette même figure 7 la courbe expérimentale décrivant l'évolution temporelle de $\Delta[\mathrm{O}]$ dans le cas d'une épaisseur de la couche d'or $x=300 \AA$ [8]. Nous constatons ainsi que, pour un temps compris entre 30 et $10^{4}$ min., $\Delta[\mathrm{O}]$ varie de $10^{18}$ à $2,8 \times 10^{19} \mathrm{at} / \mathrm{m}^{2}$ alors que le nombre de charges élémentaires neu-

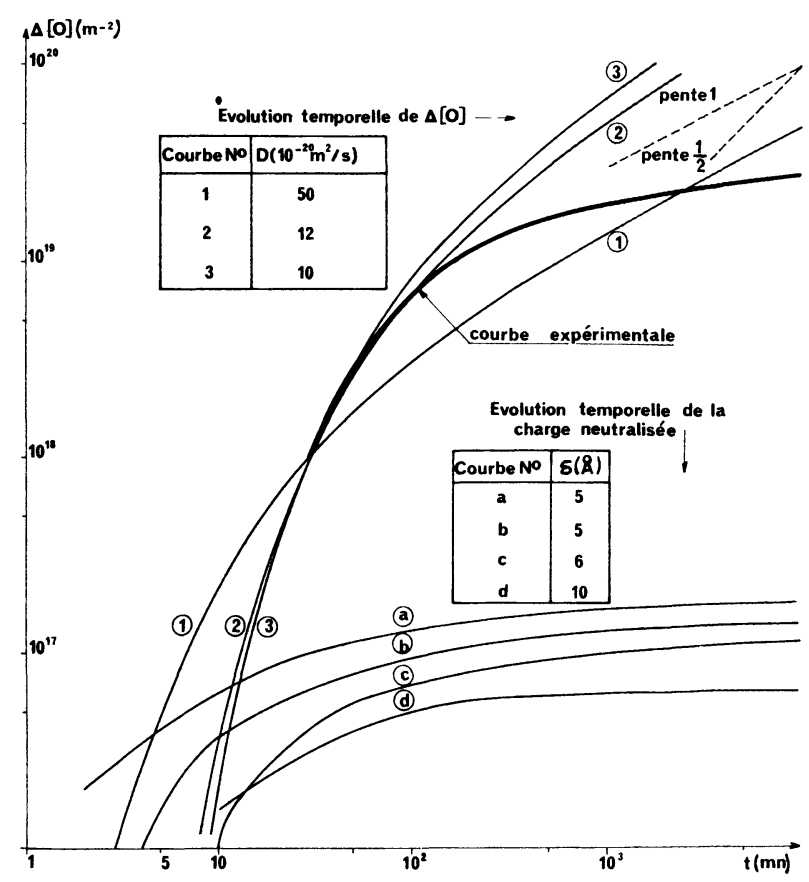

Fig. 7. - Evolutions temporelles comparées de la concentration d'oxygène en excès à l'interface $\Delta[\mathrm{O}]$ et de la charge neutralisée.

[Time evolution of the excess oxygen concentration $\Delta[\mathrm{O}]$ at the interface and of the neutralized charge.]

tralisées est de l'ordre de $10^{17} / \mathrm{m}^{2}$. Ceci montre que seule une faible partie de l'oxygène présent à l'interface participe au processus de neutralisation, probablement selon une réaction chimique du type :

$$
\mathrm{e}+\mathrm{O}+\mathrm{D}^{+} \rightleftarrows \mathrm{DO} \text {; }
$$

$\mathrm{D}^{+}, \mathrm{O}, \mathrm{e}$ désignant respectivement les charges positives (défauts), les atomes d'oxygène et les électrons (de la bande de conduction du semiconducteur, comme il sera montré dans ce qui suit).

Nous constatons que le processus de neutralisation commence quelques minutes après la mise à l'air des échantillons, pour des temps inférieurs à $30 \mathrm{~min}$. En admettant que l'évolution temporelle de la concentration d'oxygène en excès suive une loi de diffusion, nous pouvons extrapoler la courbe expérimentale représentant la variation de $\Delta[\mathrm{O}]$ pour obtenir la concentration d'oxygène atteignant l'interface au début du processus de neutralisation (voir annexe A.1). Celle-ci étant de l'ordre de $10^{16} \mathrm{at} / \mathrm{m}^{2}$, il en résulte que tout l'oxygène en excès à l'interface doit être actif en début de neutralisation, contrairement à ce qui se passe par la suite. Nous attribuons cette diminution de l'activité de l'oxygène à un effet de piégeage, lié à l'évolution de la barrière de surface du semiconducteur. Les atomes d'oxygène pourraient se comporter, en effet, comme des pièges pour les électrons de la bande de conduction (éq. (3.4)). En début de processus et en l'absence de barrière de surface, tous ces atomes sont ionisés en raison de l'abondance des électrons dis- 
ponibles dans la bande de conduction, ou en d'autres termes, parce qu'ils introduisent un niveau nécessairement situé en dessous du niveau de Fermi du semiconducteur. L'établissement progressif de la barrière de surface entraînant la courbure des bandes d'énergie vers le haut, le taux d'ionisation de l'oxygène diminue et donc sa capacité de neutraliser les défauts.

\section{Remarques :}

1) L'absence de barrière métal semiconducteur se maintenant aussi longtemps que les échantillons restent sous vide, on doit exclure tout effet de désorption d'hydrogène à travers la couche métallique ou de migration d'ions positifs vers le métal sur l'évolution ultérieure de la barrière une fois la diode mise à l'air [12].

2) D'une manière générale nos travaux en cours montrent que l'oxygène joue un rôle primordial sur le redressement et le vieillissement des structures Schottky tant sur le silicium de type $\mathrm{N}$ que de type $\mathrm{P}$. Son influence est toutefois limitée par la facilité avec laquelle il peut diffuser à travers le contact redresseur : sa constante de diffusion est en effet beaucoup plus élevée pour les métaux faiblement oxydables (or par exemple) que pour les métaux qui s'oxydent facilement (tels que l'aluminium).

4. Conclusion. - Dans cet article nous avons montré le rôle fondamental de l'oxygène dans la formation de la barrière or-silicium. Il est encore trop tôt pour proposer un modèle théorique complet permettant de suivre rigoureusement toute l'évolution de la structure. Des expériences complémentaires telles les mesures de la capacité et des pertes de la structure en fonction de la polarisation et de la fréquence devraient nous permettre d'affiner le modèle. Néanmoins, nous avons démontré malgré la séparation artificielle en deux phases, que l'évolution de la barrière est en fait un processus continu, en ce sens que tout au long de son établissement le piégeage des électrons de conduction par l'oxygène joue un rôle fondamental.

Annexe A1. - Voici les équations décrivant l'évolution temporelle de la concentration $\Delta[\mathrm{O}]$ selon une loi de diffusion [13] :

$$
\frac{C(x, t)}{C_{\mathrm{s}}}=1-\frac{\sqrt{2}}{\pi} \int_{0}^{x / 2 \sqrt{D t}} \mathrm{e}^{-\alpha^{2}} \cdot \mathrm{d} \alpha
$$

$C_{\mathrm{s}}$ est la concentration à l'origine $(x=0) \mathrm{en}^{-3}$. $C(x, t)$ est la concentration au plan d'abscisse $x$ en fonction du temps. $D$ désigne la constante de diffusion.

A l'aide de l'équation (A1.1) on déduit le flux d'oxygène $\varphi(x, t)$ en $\mathrm{m}^{-2} \mathrm{~s}^{-1}$ :

$$
\frac{\varphi(x, t)}{C_{\mathrm{s}}}=-\frac{D}{C_{\mathrm{s}}} \frac{\partial C(x, t)}{\partial t}=\frac{D}{\sqrt{\pi D t}} \mathrm{e}^{-x^{2} / 4 D t} .
$$

La quantité $\Delta[O]$ (exprimée en $\mathrm{m}^{-2}$ ) est alors déduite de l'expression précédente du flux (éq. (A1.2)) :

$$
\frac{\Delta[\mathrm{O}](t)}{C_{\mathrm{s}}}=\int_{0}^{t} \frac{\varphi(x, t)}{C_{\mathrm{s}}} \mathrm{d} t=\frac{x}{\sqrt{\pi}} \int_{0}^{2 \sqrt{D t} / x} \mathrm{e}^{-1 / \alpha^{2}} \mathrm{~d} \alpha .
$$

La fonction $\Delta[\mathrm{O}]$ a été calculée numériquement à l'aide de l'équation (A1.3) pour $x=300 \AA$ (épaisseur de la couche d'or, figures $1 a$ et $1 b$ ) et en considérant $D$ comme paramètre (Fig. 7). Nous constatons que pour $D \simeq 10^{-19} \mathrm{~m}^{2} / \mathrm{s}$ les valeurs théoriques sont en accord avec les valeurs expérimentales pour

$$
30 \mathrm{~min} . \leqslant t \leqslant 10^{2} \mathrm{~min} \text {. [8] . }
$$

Pour des temps supérieurs à $10^{2}$ min., la concentration expérimentale est inférieure à la valeur calculée à partir de l'équation (A1.3). Ceci doit être attribué à la croissance de l'épaisseur de la couche isolante entraînant un freinage du processus de diffusion.

Annexe A2. - Nous présentons ici les valeurs numériques de divers paramètres physiques pris en compte pour la modélisation de la structure MIS étudiée ; nous avons utilisé le système d'unités S.I.

Charge élémentaire : $q=1,6 \times 10^{-19} \mathrm{C}$.

Température : $300 \mathrm{~K}$.

Densité d'impuretés de type donneur : $10^{20} / \mathrm{m}^{3}$.

Coefficient de diffusion des minoritaires :

$$
1,25 \times 10^{-3} \mathrm{~m}^{2} / \mathrm{s} .
$$

Longueur de diffusion des minoritaires : $5 \times 10^{-5} \mathrm{~m}$. Longueur du semiconducteur : $3 \times 10^{-4} \mathrm{~m}$.

Mobilité des majoritaires : $0,13 \mathrm{~m}^{2} /(\mathrm{s} . \mathrm{V})$.

Permittivité de l'isolant : $35,37 \mathrm{pF} / \mathrm{m}$.

Permittivité du silicium : 106,1 $\mathrm{pF} / \mathrm{m}$.

Vitesse de collecte des électrons : $5 \times 10^{4} \mathrm{~m} / \mathrm{s}$.

Vitesse de collecte des trous : $5 \times 10^{4} \mathrm{~m} / \mathrm{s}$.

Différence des travaux de sortie entre le métal et le semiconducteur : $0,51 \mathrm{eV}$. 


\section{Bibliographie}

[1] Viktorovitch, P., Kamarinos, G. et Even, P., C.R. Hebd. Séan. Acad. Sci. 283B (1976) 119-122.

[2] Viktorovitch, P., Kamarinos, G., J. Appl. Phys. 48 (1977) 3060.

[3] Viktorovitch, P., Kamarinos, G., Even, P. et Fabre, E., accepté pour publication en 1978 dans Physica Status Solidi(a).

[4] Viktorovitch, P., Pananakakis, G., Kamarinos, G. et Basset, R., Proceedings of the : a) Photovoltaic Solar Energy Conf., Luxembourg, pp. 937-945 (Sept. 1977) b) International Electron Devices Meeting (IEDM), pp. 62-62C, Washington (décembre 1977).

[5] Ponpon, J. P., Stuck, R., Siffert, P., 12th IEEE Photovoltaic Specialists Conference, Bâton-Rouge (1976) 900.

[6] Siffert, P., Coche, A., IEEE Trans. Nucl. Sci., NS 11 (1964) 244.
[7] BADER, R., Kalbitzer, S., Z. Naturforsch. 21a (1966) 1072.

[8] PonPon, J. P., Siffert, P., Accepté pour publication à J. Appl. Phys. (1977).

[9] Ponpon, J. P., Grob, J. J., Grob, A., Stuck, R., Siffert, P., 3rd Intern. Conf. on Ion Beam Analysis, Washington D.C. USA (1977). To be published in Nucl. Instrum. Methods.

[10] Van Der Meulen, Y. J., J. Vac. Sci. Technol. 11 (1974) 985.

[11] Pananakakis, G., Viktorovitch, P. et Kamarinos, G., C.R. Hebd. Séan. Acad. Sci. 284B (1977) 471-474.

[12] TURNer, M. J., RhODERICK, E. H., Solid State Electron. 11 (1968) 91.

[13] Grove, A. S., Physique et Technologie des dispositifs à semiconducteurs (Dunod Paris) 1971. 